\title{
Les Métaphores conflictuelles dans la création de concepts et de termes
}

\section{Conflictual metaphors in conceptual and terminological creation}

Michele Prandi

Université de Gênes

Résumé

Comme les lexèmes du lexique naturel, les termes de spécialité sont par définition cohérents avec les concepts qu'ils dénotent et les objets auxquels ils se réfèrent. C'est peut être la raison qui a amené les terminologues à privilégier, parmi les métaphores documentées en terminologie, les attestations cohérentes, à savoir les catachrèses et les extensions motivées par des concepts métaphoriques conventionnels et partagés. L'hypothèse explorée dans cet essai est que les combinaisons conflictuelles de concepts, les mêmes qui sont à la base de la création poétique, jouent un rôle essentiel dans la formation de concepts, et par là de termes, métaphoriques.

Mots-clés : terme métaphorique, métaphore cohérente, catachrèse, concept métaphorique, métaphore conflictuelle, projection.

Abstract

Like lexemes belonging to natural languages, terms are by definition consistent with the concepts and objects they refer to. This is probably why terminologists tend to highlight consistent metaphors - namely, catachresis and extensions motivated by shared and conventional metaphorical concepts. The hypothesis argued for in this paper is that conflictual combinations of concepts, the same that feed poetic creations, play an essential role in creating metaphorical concepts and terms.

Keywords: metaphorical term, consistent metaphor, catachresis, metaphorical concept, conflictual metaphor, projection.

Cet article est dédié à la mémoire de Fabrizio Frasnedi

Dans l'histoire de la pensée, la métaphore a été définie à tour de rôle comme transfert d'un mot (Aristote, Poétique) et comme extension de sens d'un mot (Dumarsais 1730 [1988]), comme substitut (Fontanier 1968 ; Genette 1968 ; Groupe $\mu 1970$; Todorov 1970) et comme interaction conceptuelle (Richards 1936 ; Black 1954 ; 1967), comme système de concepts au service de la pensée cohérente 
(Blumenberg 1949; Weinrich 1958; 1964 ; Lakoff et Johnson 1980 ; Lakoff \& Turner 1989; Gibbs 1994) et comme interprétation discursive d'un signifié incohérent (Weinrich 1963, 1967 ; Prandi 1992).

Cette variété extrême, qui inclut même des types opposés, pose un problème sérieux à une définition. Toutes les définitions que nous avons envisagées sont appuyées par un nombre significatif de données. En même temps, toutes sont démenties par d'autres données. La combinaison verser l'argent, par exemple, est cohérente, alors que la combinaison verser la lumière - Le soleil versait à grands flots sa lumière sur le Mont Blanc (H. B. de Saussure) - est conflictuelle. Quand il est appliqué à l'argent, le verbe verser acquiert un signifié nouveau. Quand il est appliqué à la lumière, par contre, le même verbe ne change pas son signifié : c'est précisément la combinaison conflictuelle entre verser et la lumière qui déclenche une interprétation métaphorique. Encore, il y a des cas où une métaphore peut être envisagée comme le substitut d'une contrepartie cohérente, comme quand les gouttes de pluies sont appelées larmes (lagrime di pioggia, G. Pascoli) ; cependant, certaines métaphores n'admettent pas d'être remplacées : La lune rêve (Baudelaire), par exemple, dépeint un procès qui n'admet pas de formulation cohérente.

Ce que toutes les définitions envisagées ont en commun, c'est de focaliser l'un des résultats d'un processus métaphorique. Or, les issues d'un processus métaphorique sont nombreuses, différenciées et souvent même opposées. Si nous focalisons les issues, une définition est condamnée à être ou partielle ou fausse. Une définition de la métaphore comme extension de signifié, par exemple, est partielle si elle exclut les métaphores vives comme verser la lumière, et fausse si elle les inclut.

Face à cette impasse, il n'y a qu'une voie de sortie: déplacer l'objet de la définition des issues du procès métaphoriques, qui sont multiples, vers l'origine, qui est une. Si l'on focalise l'origine, la métaphore devient un phénomène unitaire et cohérent. Toutes les métaphores naissent du transfert d'un concept dans un domaine conceptuel étranger; le transfert provoque une interaction entre les concepts hétérogènes entraînés ; cette interaction s'ouvre à un éventail d'issues multiples et même opposées, ce qui justifie la richesse des types de métaphores attestées (Prandi 2010).

Parmi les issues hétérogènes du transfert métaphorique et de l'interaction, nous nous concentrerons sur trois types qui s'avéreront pertinents pour situer les métaphores actives en terminologie : la catachrèse, l'extension de signifié motivée par un concept métaphorique partagé et cohérent, et la construction linguistique d'un signifié complexe conflictuel

1. Métaphores cohérentes et métaphores conflictuelles

Les métaphores cohérentes se partagent entre les catachrèses (Dumarsais 1730 [1988]) et les concepts métaphoriques partagés (Lakoff et Johnson 1980 ; mais déjà Blumenberg1960 et Weinrich 1958 ; 1964).

Une catachrèse lexicale est une extension de signifié d'un mot qui est en même temps isolée et non productive. Le nom souris, par exemple, est utilisé dans une acception métaphorique pour se référer à un accessoire de l'ordinateur. Cette 
extension demeure isolée : dans le domaine des ordinateurs, on n'a pas un 'système souris' actif dans la génération d'autres extensions corrélées.

En présence de concepts métaphoriques partagés, par contre, le transfert n'est pas isolé mais il entraîne deux domaines d'expérience. Un concept métaphorique comme UNE EMOTION EST UNE PERSONNE CHERIE, par exemple, organise à partir du domaine source, à travers un réseau de projections, tout un réseau de concepts situés dans le domaine cible, comme en témoigne la présence d'extensions lexicales non isolées mais interconnectées. D'une part, un rêve peut être entretenu, nourri, soigné, caressé ; d'autre part, ces verbes s'appliquent aussi à d'autres sentiments, comme par exemple le désir ou l'espoir.

Les métaphores conflictuelles naissent en dehors du territoire de la cohérence grâce au pouvoir actif de mise en forme des expressions linguistiques. Les structures syntaxiques du noyau de la phrase ont la force de connecter les concepts atomiques non seulement dans des relations cohérents, mais aussi dans des relations conflictuelles (Husserl 1901[1970]) ; Black 1954; 1967 ; Weinrich 1963; 1967 ; Prandi 1992 ; 2004 ; 2012). S'il est cohérent de verser des substances concrètes liquides comme le vin, et même des substances liquides dans une acception métaphorique comme l'argent, par exemple, il n'est pas cohérent de verser la mélancolie des soleils couchants ou l'espoir :

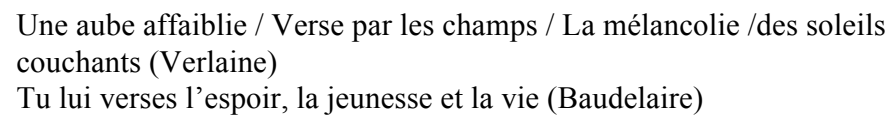

L'intérêt des expressions conflictuelles pour l'étude linguistique est évident : l'incohérence du contenu n'a pas la force de démanteler le réseau de relations grammaticales solidement implanté dans le noyau de la phrase. Si un concept cohérent est simplement porté à l'expression par une forme linguistique, un concept conflictuel est réellement construit par cette forme : on peut le concevoir comme le signifié d'une expression, et donc comme une structure sémantique, mais pas comme une structure conceptuelle indépendante. Grâce à leur capacité de connecter les concepts indépendamment de la cohérence, les structures syntaxiques du noyau de la phrase sont des instruments de création; la métaphore conflictuelle n'est que l'attestation extrême de ce pouvoir de création.

\section{Métaphores et terminologies}

La métaphore créatrice n'est pas confinée au monde ludique de la poésie mais joue un rôle de premier plan dans la formation de l'univers des concepts cohérents et partagés. On sait depuis longtemps qu'un grand nombre de concepts et de termes appartenant au lexique de la philosophie, des sciences et des savoirs spécialisés sont d'origine métaphorique (Blumenberg 1960 ; Hesse 1965[1966] ; Boyd 1979; Kuhn 19932). Ce qui est moins mis en avant, c'est le rôle des métaphores conflictuelles dans la formation et l'expression de concepts cohérents des lexiques de spécialité.

A la différence des philosophes et des épistémologues, les terminologues et les linguistes qui ont traité la question des métaphores en terminologie n'ont pas pensé d'abord aux métaphores créatrices, mais plutôt aux métaphores cohérentes: les 
catachrèses (Oliveira 2009) et les concepts métaphoriques cohérents et partagés (Temmermann 2000).

A la base de cette tendance, on trouve plusieurs raisons enracinées dans l'histoire de la discipline. En premier lieu, le conflit est immédiatement associé aux métaphores poétiques, et l'on a du mal à le voir comme un instrument de création de concepts cohérents et, à plus forte raison, de termes. Ensuite, malgré la tendance à emphatiser la distance entre signes naturels et termes, il était presqu'inévitable de penser que les instruments de l'extension lexicale - la catachrèse et le concept métaphorique partagé - étaient également les procédés actifs dans la création de termes. Une dernière raison est à chercher dans la perspective sur l'objet qui caractérise la tradition de la recherche terminologique. Si les épistémologues s'intéressent davantage aux concepts qu'aux étiquettes, dans la tradition de la terminologie l'idée dominante voit dans le terme une forme d'étiquetage d'un concept indépendant. De ce fait, les terminologues tendent à mettre à l'avant-plan les termes, et donc les métaphores, qui se limitent à étiqueter des classes d'objets indépendants, et font passer à l'arrière-plan les concepts, et donc les métaphores qui agissent sur la structure des concepts.

Or, toutes ces motivations sont susceptibles d'être critiquées. La présence d'un conflit n'est pas un obstacle insurmontable dans le processus de la création de concepts cohérents ; au contraire, le conflit est l'un des instruments de création de concepts, et donc de termes, par excellence, comme le montrent les travaux des épistémologues cités plus haut. Nous reviendrons sur ce point. Ensuite, entre la formation des signes naturels et la création des termes il y a une différence essentielle, qui empêche de transférer dans le domaine de la terminologie les hiérarchies valables pour les lexiques des langues naturelles. Dans les lexiques naturels, la création des concepts - des signifiés - aussi bien que la création des signifiants, est un processus essentiellement anonyme. En terminologie, par contre, il y a peu de processus anonymes. La phase de stabilisation des concepts, qui prépare le partage et la cohérence, est l'issue d'une discussion explicite. Mais c'est surtout le geste créateur destiné à générer un concept qui est déclenché par un acte de création individuel semblable à celui du poète. Cela dit, la catachrèse et, surtout, l'action des concepts métaphoriques partagés, sont en mesure de justifier l'essentiel de la création lexicale dans les langues naturelles, où la place réservée à la créativité individuelle est marginale, mais pas en terminologie, où la création individuelle est primordiale ${ }^{1}$. Or, il est clair que la création individuelle ne saurait être justifiée ni à partir d'un système de concepts métaphoriques préalablement donnés et partagés, ni, à plus forte raison, à partir de catachrèses isolées - elle a besoin de passer par la construction linguistique de combinaisons conflictuelles de signifiés. Finalement, en terminologie, comme en épistémologie, il faut tout d'abord se demander si la création se limite au terme ou si elle concerne le concept lui-même, et cela vaut à plus forte raison pour la création métaphorique. La catachrèse souris est un exemple de création limitée au terme, car elle se limite à coller une étiquette métaphorique sur un concept indépendant qui correspond en extension à une classe d'objets. Tout

\footnotetext{
${ }^{1}$ Le rôle de la création individuelle dans la formation des termes n'exclut évidemment pas que ses résultat soit normalement discutés, corrigés, entérinés, rejetés, soit par la communauté des experts, soit par des institutions officielles de l'Etat.
} 
autre terme, métaphorique ou pas, aurait pu fonctionner aussi bien. Lorsqu'on parle de circulation en physiologie ou en économie (Rossi 2015 : 65-69) ou de code génétique, par contre, la métaphore sculpte le concept, pour ainsi dire, avant de lui donner un nom ${ }^{2}$. La métaphore n'est pas un instrument d'étiquetage, de pure et simple création d'un mot, mais l'instrument de création d'un concept. Cette caractéristique distingue nettement les métaphores créatrices, constitutives de concepts, des métaphores pédagogiques, d'illustration ${ }^{3}$. Alors que les métaphores créatrices structurent un concept, l'utilisation de métaphores pédagogiques présuppose l'accessibilité indépendante d'un concept solidement établi, qui a déjà son nom. Sur ce point, la métaphore pédagogique s'approche du pur et simple étiquetage, à une différence prêt : à la différence de l'étiquetage, les métaphores pédagogiques sont essentiellement des substituts.

Toutes ces raisons poussent à accorder aux métaphores créatrices issues de combinaisons conflictuelles de concepts un rôle de premier plan dans la création de concepts et de termes.

\section{A l'origine de la création : la pression du conflit conceptuel}

L'observation des expressions conflictuelles nous conduit aux racines de la création. Dans une expression conflictuelle, un foyer (focus, Black 1954) étranger s'insère dans un cadre cohérent avec le texte ou le discours (frame, Black 1954). Dans la phrase Votre âme est un paysage choisi (Verlaine), votre âme est le cadre cohérent dans lequel le foyer paysage introduit un concept étranger : l'énoncé parle de l'âme de l'interlocuteur et non pas d'un paysage. Les concepts qui entrent en conflit sont la teneur (Richards 1936), cohérente avec le cadre, et le sujet de discours subsidiaire (Black 1954), qui est incohérent. Dans notre exemple, la teneur coïncide avec le cadre - l'âme - alors que le sujet subsidiaire coöncide avec le foyer ${ }^{4}$ : le paysage.

Le conflit produit une pression conceptuelle qui est prête à se décharger ou bien sur le foyer étranger ou bien sur le cadre cohérent. Etant donné une expression conflictuelle comme La lune rêve (Baudelaire), par exemple, on peut imaginer soit une pression du cadre cohérent sur le foyer incohérent, dont l'issue extrême est la substitution - la lune, par exemple, est voilée de brouillard - soit une pression du foyer sur la teneur: sous la pression du foyer rêver, par exemple, la lune est vue

\footnotetext{
${ }^{2}$ Il y a même des cas où le processus métaphorique conduit à la création d'un concept sans pourtant lui transmettre le nom du modèle. Par exemple, beaucoup de témoignages appuient l'hypothèse que le concepts d'atome a été inspiré à Leucippe et Démocrite par les lettres de l'alphabet grec (Hallyn 2000), mais aucune trace de cette origine demeure dans le nom qui s'est imposé depuis Démocrite.

${ }^{3}$ La distinction entre métaphores constitutives et métaphores d'illustration est explcitèe par Boyd (19932: 359-360).

${ }^{4}$. La coïncidence de cadre et teneur et de foyer et sujet subsidiaire ne vaut que pour l'emploi métaphorique du nom en position prédicative. Dans le nom référentiel, par exemple, la teneur, tout en étant cohérente avec le cadre, ne coïncide pas avec lui, mais s'oppose au foyer dans une corrélation paradigmatique in absentia. Dans l'expression Jean a marié un rossignol, où le foyer un rossignol se réfère à une femme, la teneur n'est pas le cadre Jean a marié mais le référent visé - la femme - cohérent avec le cadre.
} 
comme un être humain. Parmi les propriétés qui distinguent les métaphores cohérentes et les métaphores conflictuelles, la plus pertinente pour notre question est précisément l'orientation opposée de la pression conceptuelle qui se déclenche quand deux concepts hétérogènes sont connectés.

En présence de métaphores cohérentes, c'est le concept étranger - le foyer - qui s'adapte à la pression de la teneur. Dans la connexion verser l'argent, motivée par le concept métaphorique partagé d'argent liquide, par exemple, le verbe verser adapte son signifié au concept d'argent pour lui faire place. Cette adaptation éteint le conflit et confirme les structures conceptuelles conventionnelles et acquises.

En présence de métaphores conflictuelles, la pression du concept étranger - du foyer - pousse à restructurer le concept cohérent : la teneur. Dans la connexion verser la lumière, le verbe verser - le foyer - garde son signifié et décharge la pression du conflit sur la teneur, qui voit son profil conceptuel restructuré : la lumière se change en substance liquide. C'est ce renversement de la pression conceptuelle qui change le conflit en instrument de création : si la pression sur le foyer garantit l'identité acquise des concepts, la pression sur la teneur la remet en question.

Si nous passons à la création de concepts de spécialité et des termes qui les désignent, il est clair que la première phase de la mise en œuvre d'un concept réellement novateur ne peut que partager avec la métaphore poétique l'orientation de la pression conceptuelle, qui investit la teneur: le concept nouveau ne peut se former que sous la pression d'un concept étranger, en quittant le terrain solide des concepts acquis. C'est seulement dans une phase successive, de stabilisation, que la mise au point du nouveau concept emprunte un chemin divergent par rapport à la métaphore poétique pour atteindre la cohérence et le partage par la communauté des experts.

4. La création de concepts grâce au conflit

4.1. L'élaboration créatrice d'un concept métaphorique cohérent: la valeur linguistique chez Saussure

A la différence d'une catachrèse, un concept métaphorique cohérent et partagé n'est pas isolé mais projectif. En même temps, le potentiel de projection est sévèrement limité par rapport à ce qui arrive en présence de métaphores conflictuelles, car seulement une partie des inférences autorisées par la source est pertinente pour la catégorisation partagée de la cible et attestée dans le lexique d'une langue. En français, par exemple, l'idée que l'argent est liquide autorise à penser qu'on peut le verser, le prélever, l'utiliser pour arroser quelqu'un, mais pas qu'il forme des gouttes, des flaques ou des vagues. Tant qu'il est employé dans les limites du partage social, et donc dans les limites d'un lexique naturel, un concept métaphorique ne met pas en discussion notre paysage conceptuel hérité et aucun effort cognitif n'est demandé pour son emploi. Toutefois, grâce à sa nature projective, un concept métaphorique offre un réservoir d'inférences potentielles qui peuvent être explorées pour lancer des idées nouvelles par-delà le seuil du partage social sanctionné par les structures lexicales. A ce moment, il se déclenche un processus qui, à la différence de la circulation anonyme de lexèmes, demande 
l'œuvre d'un démiurge : individuel au moment de la création, et collectif, coïncidant avec la communauté des experts, au moment de la mise au point du concept cohérent.

Un exemple significatif de valorisation créatrice du potentiel latent dans un concept métaphorique cohérent et partagé est l'application du concept de valeur aux signes linguistiques dans le Cours de Saussure (1916[1972]).

L'emploi du mot valeur appliqué aux signes linguistiques est redevable d'un concept métaphorique cohérent et partagé - le concept de mot comme monnaie dont l'histoire dans la culture occidentale est retracée par Weinrich (1958). Comme le fait remarquer Weinrich, l'idée que les mots ont une valeur, active depuis toujours dans la culture occidentale, est un concept actif et productif, qui structure un véritable "champ métaphorique». Dans les siècles, ce concept a alimenté tant l'emploi linguistique commun de plusieurs communautés que l'élaboration créatrice des poètes et des philosophes. En français, par exemple, on parle de trésor, de banque de mot, de richesse ou pauvreté lexicale; en italien et en anglais, on applique les verbes coniare et coin (frapper) à la néologie. Par ailleurs, Goethe parle de «lettres d'échange poétiques», et les philosophes sceptiques se servent de la métaphore pour appuyer l'idée de la nature sociale et non naturelle de la langue (Weinrich $1958: 37$ ).

Quand Saussure emprunte le mot valeur pour en faire un terme de la linguistique, il se comporte davantage comme le poète et le philosophe que comme un usager spontané de la langue. Il ne se limite pas à exploiter une inférence acquise dans le cadre du concept partagé - notamment l'idée qu'un mot peut être échangé contre un contenu - mais, qui plus est, il développe une idée également autorisée par la métaphore de base mais située en dehors de l'éventail d'inférences partagées. La valeur d'une monnaie ne se réduit pas à la dimension externe - à ce qu'on peut acheter avec elle - mais inclut une dimension interne : la valeur émane d'un système monétaire. D'une manière similaire, la valeur d'un mot ne se réduit pas à la signification, mais inclut la dépendance d'un système: les unités linguistiques de tous niveaux sont des «valeurs émanant du système »; «Dans l'intérieur d'une même langue, tous les mots qui expriment des idées voisines se limitent réciproquement » (Saussure (1916[1972: 160;162]).

Le concept métaphorique cohérent et partagé est caractérisé par une identité double: il autorise la mise au point d'idées créatrices dans le cadre d'une conceptualisation partagée. Grâce à cela, il se prête aussi bien à la création de concepts, comme le montre l'exemple de Saussure, qu'au simple étiquetage, comme le montrent des termes comme greffe ou trapianto dans le lexique médical, ou, dans le domaine de l'informatique, tous les termes issus de la métaphore de l'écran comme bureau.

\subsection{La création issue du conflit : l'idée de sélection naturelle}

La création fondée sur une combinaison conflictuelle de concepts est plus radicale que la simple élaboration d'inférences créatrices à partir de concepts métaphoriques acclimatés dans notre culture. Elle ne se limite pas à tirer des inférences créatrices de 
concepts connus, mais crée des combinaisons conceptuelles inattendues et surprenantes dans le but précis de solliciter des inférences inexplorées.

Cette forme radicale de création se développe en deux phases: une pars destruens, qui défie l'identité acquise des objets, et une pars construens, qui redessine le profil des mêmes objets d'après des modèles nouveaux. La métaphore conflictuelle est l'instrument idéal pour les deux tâches : le conflit et la pression conceptuelle qu'il déclenche défient l'identité acquise des concepts ; le transfert et la projection les redessinent sous la pression de concepts étrangers. Paivio (1979: 50) illustre cette idée avec une métaphore : « metaphor is a solar eclipse. It [...] obscures its [de «l'objet d'étude »] literal and commonplace aspects while permitting a new and subtle understanding to emerge ${ }^{5} »$.

Les métaphores conflictuelles valorisent des concepts radicalement étrangers comme autant de voies d'accès épistémique (Boyd 1979[1993: 377]). De ce fait, elles se prêtent davantage à l'idéation de nouveaux concepts dans les phases de 'changement de paradigme' qu'à l'illustration et à la divulgation des acquisitions d'une 'science normale' (Kuhn 1962). Ces métaphores «constitute, at least for a time, an irreplaceable part of the linguistic machinery of a scientific theory ${ }^{6}$ ", dépourvues d'une paraphrase non métaphorique (Boyd 1979[1993: 360).

Le concept de 'sélection naturelle' (Darwin 1859[1950]) est un bon exemple de concept né d'une combinaison conflictuelle. La sélection est une action humaine accomplie par un agent à la poursuite d'un but intentionnel : le modèle projeté sur la nature inanimée est «l'agriculteur clairvoyant» qui sélectionne son bétail et contrôle sa reproduction pour améliorer sa qualité : «Can the principle of selection, which we have seen is so potent in the hands of man, apply in nature?», est la question de Darwin (1859[1950: 69]). La pars destruens du projet déconstruit l'idée d'un Dieu onnipuissant qui travaille réellement à la réalisation d'un projet. La pars construens projette le modèle de l'agriculteur clairvoyant sur la nature vivante à la recherche d'un principe impersonnel fonctionnellement équivalent: " If a being infinitely more sagacious than man (not an omniscient creator) during thousands and thousands of years were to select all the variations which tended toward certain ends $[\ldots]^{7} »$ (Darwin 1842[1958: 45]), un tel être, donc, de quelle façon peut on penser qu'il agirait ? La réponse est l'idée de sélection naturelle poussée par la lutte pour la survie, qui met en compétition tant les êtres de la même espèce que les espèces ellesmêmes :

"It may be said ${ }^{8}$ that natural selection is daily and hourly scrutinizing, throughout the world, every variation, even the slightest; rejecting that which

\footnotetext{
5. «La métaphore est une éclipse solaire. Elle obscurcit les aspects routiniers des objets d'étude permettant l'émergence d'une compréhension nouvelle et plus subtile ».

${ }^{6}$. Les métaphores constitutives « représentent, du moins pour un certain temps, un ingrédient irremplaçable de la machinerie linguistique d'une théorie scientifique ».

${ }^{7}$. Le texte de cette première ébauche contient quelques lacunes syntaxiques, parmi lesquelles figure l'apodose de ce contrefactuel, qui cependant peut être facilement inférée.

8. Comme la forme conditionnelles de la citation précédente, le hedge bloque la personnification et montre que Darwin est conscient de la fonction de la métaphore en tant que premier pas ver un concept cohérent. Voir aussi des précisions comme la suivante : «I
} 
is bad, preserving and adding up all that is good; silently and insensibly working, whenever and wherever opportunity offers, at the improvement of each organic being in relation to its organic and inorganic conditions of life" (Darwin 1859[1950: 72]).

\subsection{La stabilisation des métaphores conflictuelles créatrices de concepts}

Le processus de création conceptuelle basé sur le conflit met en discussion notre paysage conceptuel hérité : la nature n'est pas un agriculteur clairvoyant. A la différence de ce qui se passe avec les concepts métaphoriques cohérents, il n’y a rien de partagé au préalable dans la métaphore. Son contenu est totalement à inférer à partir du conflit, et donc à élaborer et à discuter : par le créateur d'abord, et puis de la part de la communauté des savants, dans un travail qui continue.

Le critère de la pression conceptuelle rapproche la création de concepts par le conflit aux métaphores vives de la poésie. Ce n'est pas par hasard que certaines métaphores conflictuelles sont documentées tant dans la poésie que dans la recherche scientifique. L'idée des vagues de lumière, par exemple, se trouve dans la poésie romantique anglaise comme l'une des mille incarnations du concept conflictuel de lumière liquide :

\section{It [the young moon] floats upon the sunset's sea of beams, Whose golden waves in many a purple line Fade fast (Shelley)}

Le même concept d'onde appliqué à la lumière est un pilier de la physique expérimentale de Huygens jusqu'à Maxwell. En italien et en anglais, le même mot onda, wave - couvre les deux concepts, trahissant la source unique de la métaphore (Prandi 2012). L'affinité d'élection entre la métaphore créatrice de concepts scientifiques et la métaphore poétique n'échappe pas à Hesse (1965[1966: 160]) : « It is necessary that there should be patent falsehood or even absurdity in taking the conjunction literally. Man is not, literally, a wolf; gases are not in the usual sense collections of massive particles ». Quand il est étendu pour faire place à la nature parmi ses sujets cohérents, le signifié du mot sélection n'est pas adapté au concept préexistant de nature, mais pousse à redessiner le profil du concept sous la pression d'un concept conflictuel.

A la différence des métaphores poétiques, la création de concepts est soumise à la condition de la cohérence. A la différence des emplois métaphoriques anonymes des mots, cependant, la cohérence n'est pas garantie a priori, mais elle est à conquérir par la communauté des savants avec un travail créateur.

Les emplois métaphoriques partagés des lexèmes naturels circulent dans la société comme des tautologies du lexique; en français, par exemple, il est cohérent d'embrasser une idée ou de cultiver un projet du fait que les membres de la communauté acceptent ces combinaisons. Les termes de spécialité métaphoriques, au contraire, ne sont pas acceptés comme des tautologies par la communauté des

should premise that I use the term Struggle for Existence in a large and metaphorical sense » (Darwin 1859(1950: 54)). 
experts ; au contraire, ils sont discutés et accueillis dans la mesure où les inférences qu'ils autorisent ouvrent des chemins productifs accessibles au contrôle empirique.

Il en est ainsi de l'idée de valeur comme dépendance du système. La phase d'élaboration individuelle est bientôt suivie d'une discussion et de la mise à l'épreuve du concept qui prépare son partage de la part de la communauté des savants. Appliquée aux signifiés des lexèmes, l'idée de valeur aboutit à l'idée de champ sémantique ${ }^{9}$ (Trier 1931); appliquée aux sons, à la phonologie pragoise (Troubetzkoy 1939). Et il en est ainsi de l'idée de sélection naturelle, qui, depuis un siècle et demi, ne cesse pas d'inspirer de nouvelles applications tant dans les sciences de la nature que dans les sciences de l'homme.

\section{Conclusion}

Le parcours qui nous mène de la catachrèse à la création conflictuelle en passant par les concepts métaphoriques cohérents est aussi le chemin de la créativité, s'étalant du simple étiquetage de concepts indépendants jusqu'à la conception de nouveaux concepts.

Sur la base des exemples que nous avons analysés à chaque étape, ce parcours nous a conduit en même temps de l'atelier où se forgent les dénominations les plus communes jusqu'aux sommets de création scientifique et philosophique, où naissent les concepts qui changent les destins de la recherche. Une question qui se pose tout naturellement à ce point est la suivante : cette progression est-elle due au hasard des exemples, ou ne va-t-elle jusqu'à dessiner une hiérarchie dans la création ellemême ? Notre intuition nous dit que le souffle de la création, exalté dans les grands tournants de la philosophie et des sciences, peut investir tout aussi bien la prose du quotidien.

En formulant cette intuition, nous pensons par exemple au lexique des sommeliers (Rossi 2009), qui force des mots dans des combinaisons inusuelles et potentiellement conflictuelles pour les changer, dans les mains des experts, en termes techniques: par exemple, les tanins soyeux, les jambes ou les larmes du vin... Ces termes, par ailleurs, ne se limitent pas à étiqueter des concepts connus, mais ouvrent une véritable « voie d'accès » à tout un domaine jusque là inexploré. Un autre exemple intéressant est fourni par les remarques que Fauconnier (1997: 1825) consacre à l'application au domaine informatique de termes comme virus, infection, contaminer, immune, désinfectant, vaccin : grâce à la métaphore, "We can start looking for 'disinfectants' and 'vaccines', we can think of making our system 'immune' or 'safe' before we know if this is technologically feasible, and a fortiori before the computer domain actually contains any real equivalents of 'disinfectants'

\footnotetext{
9. Cela montre que pour la métaphore constitutive l'acquisition de la cohérence et sa transformation en terme reconnu n'implique pas la désactivation de son potentiel, contrairement à ce qu'écrit Détrie (2001 : 202) : «Une métaphore conventionnelle est donc nécessairement usée, étant le produit d'une conceptualisation métaphorique consensuelle». Boyd (19932: 273) souligne que les métaphores constitutives de concepts scientifiques, comme les métaphores poétiques, ne sont pas condamnées au déclin.
} 
and 'immunity' ${ }^{\prime 10} »$. Loin d'être à la poursuite des objets comme autant d'étiquettes neutres, bien des termes forgent véritablement les concepts.

Les exemples que nous avons analysés, en définitive, montrent que la création de termes dans tous les domaines, des plus élevés jusqu'aux plus quotidiens, est ouverte à tous les types de métaphores : aux catachrèses isolées, aux concepts métaphoriques cohérents et partagés, aux métaphores conflictuelles et créatrices. Dans tous ces cas, l'équilibre entre la création de termes et la création de concepts change de façon significative. Les métaphores créatrices issues de combinaisons conflictuelles mettent au premier plan la création de concepts nouveaux; le terme n'est que la sanction de ce processus novateur. A l'extrémité opposée, la catachrèse exploite des analogies évidentes, enracinées souvent dans les propriétés immédiatement accessibles à la perception, pour créer des étiquettes qui n'ajoutent rien, ou presque, aux concepts qu'elles désignent. Au milieu, idéalement, se situent les concepts métaphoriques cohérents et partagés, qui, tels Janus, s'orientent dans deux directions opposées. D'une part, ils peuvent se limiter à exploiter des réservoirs tous prêts de concepts pour coller des étiquettes suggestives sur des concepts connus; d'autre part, ils autorisent des inférences inattendues, qui sortent du périmètre des projections acquises et conventionnelles, et, de ce fait, sollicitent la création de concepts nouveaux.

\section{Références}

ARIstote (1965), Poétique, Paris : Les Belles Lettres.

Black, M. (1954), « Metaphor », Proceedings of the Aristotelian Society 55, 273 294. Réimpr. in BLACK, M. (1962), 25-47.

Black, M. (1962), Models and Metaphors, Ithaca - Londres : Cornell University Press.

BLACK, M. (1979), « More about metaphor », in A. Ortony (éd.), Metaphor and Thought, Cambridge : Cambridge University Press, 19-43.

Blumenberg, H. (1960), Paradigmen zu einer Metaphorologie, Bonn : Bouvier und Co.

BOYD, R. $\left(1993^{2}\right)$, « Metaphor and theory change: what is 'Metaphor' a metaphor for? », in A. Ortony (éd.), Metaphor and Thought, Cambridge : Cambridge University Press, 481-532.

DARwIN, CH. (1842(1958)), «The sketch of 1842 », in Ch. Darwin \& A.R. Wallace (éds.), Evolution by Natural Selection, Cambridge : Cambridge University Press, 41-88.

DARWIN, CH. (1859(1950)), On the Origin of Species by means of Natural Selection, A Reprint of the First Edition, Londres : Watts \& Co.

\footnotetext{
${ }^{10}$. «On peut commencer à parler de désinfectants et de vaccins, et envisager de rendre des systèmes immunes avant même de savoir si cela est faisable au plan technologique et $a$ fortiori avant que le domaine des ordinateurs ne contienne des équivalents réels de ces concepts ».
} 
Detrie, C. (2001), Du sens dans le processus métaphorique, Paris : Champion.

Dumarsais, C. (1730(1988)), Des tropes, ou des différents sens, Paris: Flammarion.

FAuCONnIER, G. (1997), Mappings in Thought and Language, Cambridge : Cambridge University Press.

FontANIER, P. (1968), Les figures du discours, Paris : Flammarion. Réunit: Manuel classique pour l'étude des tropes $\left(1830^{4}\right)$ et Traité général des figures de discours autres que les tropes (1827).

Genette, G. (1966), «Introduction» à P. Fontanier, Les figures du discours, Paris : Flammarion, 5-17.

GiBbs, R. W. (1994), The Poetics of Mind, Cambridge : Cambridge University Press.

GROUPE $\mu$ (1970), Rhétorique générale, Paris : Larousse.

Hallin, F. (2000), «Atoms and letters », in F. Hallyn (éd), Metaphor and Analogy in the Sciences, Dordrecht: Kluwer, 53-69.

Hesse, M. B. (1965(1966)), « The Explanatory Function of Metaphor », in Y. BarHillel (éd.), Logic, Methodology, and Philosophy of Science, Amsterdam : North Holland, 157-177. Réimpr. in M. B. Hesse, Models and analogies in science, South Bend : University of Notre Dame Press.

Husserl, E. (1900(1961)), Logische Untersuchungen, Band 1, Halle 1900; Band II, Halle 1901. Edition critique: Husserliana, Vol. XVIII (1975) - XIX, I-II (1984), La Haye, Nijoff. Tr. Fr. (de la $2^{\mathrm{e}}$ éd., Halle, 1922-23) : Recherches logiques, Tome I, Paris 1959; Tome II, Paris, 1961-62.

Kunn, TH. S. (1962), The Structure of Scientific Revolutions, Chicago - Londres : The University of Chicago Press.

KuHN, TH. S. $\left(1993^{2}\right.$ ), « Metaphor in Science », in A. Ortony (éd.), Metaphor and Thought, Cambridge : Cambridge University Press, 533-542.

LAKoff, G., Johnson, M. (1980), Metaphors we Live by, Chicago - Londres : The University of Chicago Press. Tr. Fr. : Les métaphores de la vie quotidienne, paris : Les Editions de Minuit.

LAkoff, G. \& Turner, M. (1989), More than Cool Reason, Chicago - Londres : The University of Chicago Press.

OliveirA, I. (2009), Nature et fonction de la métaphore en science, Paris: L'Harmattan.

PRANDI, M. (1992), Grammaire philosophique des tropes, Paris : Les Editions de Minuit.

Prandi, M. (2004), The Building Blocks of Meaning, Amsterdam - Philadelphie : John Benjamins.

PRANDI, M. (2010), " L’interaction métaphorique: une grandeur algébrique », Protée 38 (1), Le Groupe $\mu$ entre rhétorique et sémiotique, 75 - 84.

PRANDI, M. (2012) : " A Plea for Living Metaphors: Conflictual Metaphors and Metaphorical Swarms », Metaphor and Symbol 27 (2), 148-170.

Richards, I. A. (1936), The Philosophy of Rhetoric, Oxford: Oxford University Press. 
Rossi, M. (2009), «L’emploi de la métaphore comme ressource pour la néologie en terminologie: le cas du langage de la dégustation du vin ", in P. Dury, F. Maniez, N. Arlin \& C. Rougemont (éds.), La Métaphore en langues de spécialité, Grenoble : Presses Universitaires de Grenoble, 199-227.

Rossi, M. (2015), In rure alieno. Métaphores et termes nomades dans les langues de spécialité, Berne : Peter Lang.

SAussure, F. DE (1916(1972), Cours de linguistique générale, Paris : Payot. Edition critique par T. de Mauro, Paris : Payot.

TEMmermann, R. (2000), Towards new ways of terminology description: the sociocognitive-approach, Amsterdam-Philadelphie : John Benjamins.

TrIER, J. (1931(1973)), Der deutsche Wortschatz im Sinnbezirk des Verstandes. Die Geschichte eines sprachlichen Feldes. Part I: Von den Anfangen bis zum Beginn des 13. Jahrhunderts. Heidelberg: Winter. Réimpr. in A. van der Lee, \& O. Reichmann (éds.), Aussätze und Vorträge zur Wortfeldtheorie, La Haye - Paris : Mouton, 40-65.

Troubetzkoy, N. S. (1939[1949]), Grundzüge der Phonologie, Prague : Travaux du Cercle Linguistique de Prague. Tr. fr.: Principes de Phonologie, Paris: Klincksieck.

WeINRICH, H. (1958), « Münze und Wort. Untersuchungen an einem Bildfeld », Romanica, Festschrift Rohlfs, Halle : Niemeyer, 508-521.

WeINRICH, H. (1963), «Semantik der kühnen Metapher », Deutsche Vierteljahrsschrift für Literaturwissenschaft and Geistesgeschichte 37, 325-344.

WEINRICH, H. (1964), «Typen der Gedächtnismetaphorik », Archiv für Begriffsgeschichte 9, 23-26.

WeINRICH, H. (1967), « Semantik der Metapher », Folia Linguistica 1, 3-17. 